\title{
THE EFFECT OF OXYGEN AND TYPE OF CONTAINER ON THE RETENTION OF THE FERTILIZING CAPACITY OF FOWL SPERMATOZOA STORED IN VITRO*
}

\author{
F. G. PROUDFOOT AND D. K. R. STEWART \\ Canada Department of Agriculture, Kentville, Nova Scotia \\ (Received 28th March 1966, revised 7th June 1966)
}

\begin{abstract}
Summary. The fertilizing capacity of fowl spermatozoa, kept up to 17 hr at $10^{\circ} \mathrm{G}$, was enhanced by storage in an atmosphere of oxygen.

The effective degree of oxygenation of the semen with respect to the survival of spermatozoa in containers of different sizes has been considered and discussed.

The fertilizing capacity of semen did not appear to be affected by a rapid fall in temperature during its collection.
\end{abstract}

\section{INTRODUCTION}

In a recent report Proudfoot (1966) presented evidence that fowl spermatozoa stored for $7 \mathrm{hr}$ at $10^{\circ} \mathrm{C}$ in an oxygen environment exhibited higher fertilizing capacity than those kept in nitrogen, carbon dioxide or air.

Schindler \& Nevo (1962) noted that, in a suspension of washed fowl spermatozoa in a sugar-free buffer, there was a narrow zone adjacent to the air-liquid interface in which the spermatozoa were motile, whereas in the greater part of the suspension they were immotile.

The present work was undertaken to study the effect on the fertilizing capacity of fowl spermatozoa of: (1) sudden versus gradual cooling before storage under oxygen, (2) oxygenation of different depths of semen in a container, (3) prolonged storage periods in an atmosphere of oxygen, and (4) bubbling oxygen through distilled water before flushing semen containers.

\section{MATERIALS AND METHODS}

Males and females of a random-bred Leghorn strain were used in eight experiments reported below. The males were handled and semen collected from them for 2 days before using semen for the experiments.

Semen was collected by the method of Burrows \& Quinn (1937) and the insemination technique used was that described by Parker, McKenzie \& Kempster (1942).

Approximately 20 min elapsed during the collection of semen from about twenty males. The semen was pooled and stirred gently before being dispensed

* Contribution No. 1235 from the Research Station, Canada Department of Agriculture, Kentville, Nova Scotia. 
into vials, which took about another $14 \mathrm{~min}$ before it was placed in storage in either air or 99 to $100 \%$ oxygen. A semen sample for each storage treatment consisted of twelve to sixteen vials from which thirty-eight to fifty females were inseminated. Each vial contained $0.4 \mathrm{ml}$ of semen, and it approximated to the output of a single collection from a Leghorn male.

Storage temperature was $10^{\circ} \mathrm{C}$ except for Experiment 5, in which it was $5^{\circ} \mathrm{C}$. Semen was again gently stirred just before insemination when $0.1 \mathrm{ml}$ of semen was inseminated into each female between 15.00 and 16.00 hours. Inseminations were completed within 10 min after the samples were removed from storage.

Four different kinds of plastic vials were used in the experiments (Plate 1). The graduated vial was a part of a centrifuge tube which had a gas-semen surface area of $50 \mathrm{~mm}^{2}$ and was used only in Experiment 2. The other three kinds of vials are described in Table 2 and were referred to as large, medium and small.

In all the experiments eggs were collected from the 2nd day following insemination, were set in an incubator on the day after they were laid and broken for a macroscopic evaluation of fertility after 3 days' incubation. The only selection of eggs for setting was to eliminate cracked, double-yolked and yolkless eggs. Data presented are based on total eggs set.

Experiment 1 was designed to study the effect of a gradual decline in semen temperature during the collection and dispensing process as opposed to a sudden drop in temperature. The gradual decline in temperature was achieved by using a water bath at $35^{\circ} \mathrm{C}$ and holding the semen at this temperature during the 20-min collection period as opposed to holding the semen at $10^{\circ} \mathrm{C}$ in air. Following the collection period all semen was dispensed into medium vials at a room temperature of $22^{\circ} \mathrm{C}$, flushed with oxygen, corked and stored for $7 \mathrm{hr}$.

Experiment 2 was designed to study the effect of the size of the storage vial when enclosed in a plastic bag; sixteen graduated and sixteen large vials were compared. Four racks, each containing eight vials, were each enclosed in a Cryovac (polyvinylidene chloride co-polymer resin) bag $(20 \times 30 \mathrm{~cm})$ which was flushed with oxygen and sealed with plastic adhesive tape. Before sealing, sufficient corks for each vial were enclosed so that the vials could be corked by manipulating them through the film before puncturing the plastic bag at the beginning of insemination. The oxygen atmosphere was thus maintained up to the time of insemination. Semen was stored for $7 \mathrm{hr}$ before insemination.

Experiment 3 was designed to study the effect of oxygen versus air under conditions where dehydration was controlled to some extent by flushing out vials and corking rather than using a Cryovac bag as in Experiment 2. Semen was dispensed into thirty-two large corked vials, one half of which contained an oxygen atmosphere and the remainder air. The semen was stored for $7 \mathrm{hr}$ before insemination.

In Experiment 4 the large vial was used with $0.4 \mathrm{ml}$ of semen dispensed into each of thirty-two vials. One half of the vials were flushed with oxygen and corked; the remaining sixteen were flushed with oxygen which had been previously bubbled through distilled water and corked. The storage period was $7 \mathrm{hr}$. 
PLATE 1

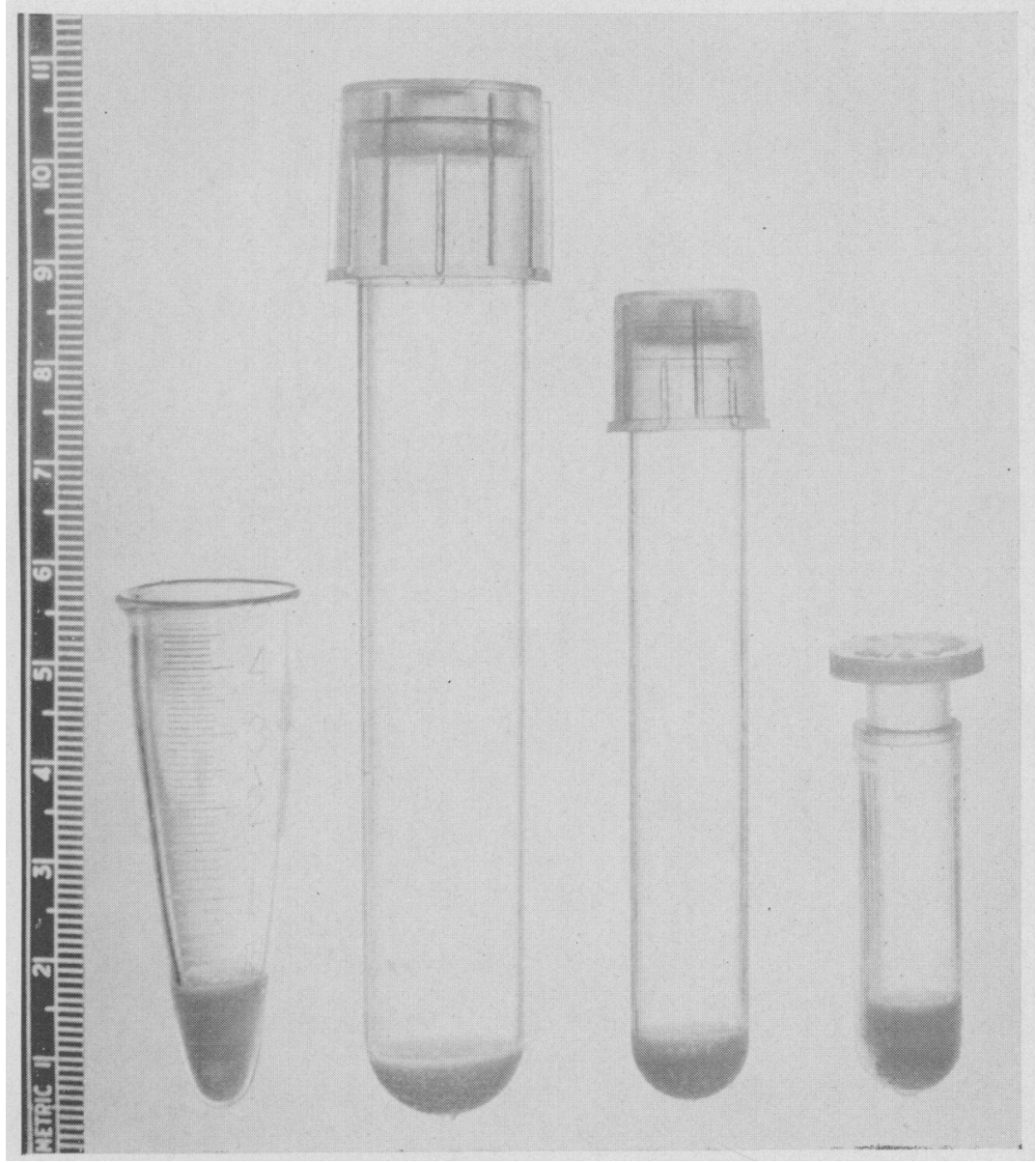

From left to right are the graduated, large, medium and small vials each containing $0.4 \mathrm{ml}$ of semen. 
Experiment 5 was designed to study the effect of storing semen in large compared with medium vials. Semen was stored at $5^{\circ} \mathrm{C}$ for $15 \mathrm{hr}$. Twelve vials of each size were used.

Experiment 6 was similar to Experiment 3 except that the storage period was extended to $15 \mathrm{hr}$ and medium vials were used.

Experiment 7 was similar to Experiment 6, except that the storage period was extended to $17 \mathrm{hr}$ and insemination was conducted at 15:00 hours compared with 16:00 hours for Experiment 6 .

Experiment 8 was designed to compare the fertility of semen stored in medium and small vials. Sixteen vials of each size were used. All vials were flushed with oxygen, corked and stored for $15 \mathrm{hr}$.

Fresh semen from similar males was inseminated at the same time as Experiments 1,2 and 3, to serve as a control for all experiments.

For statistical analysis observations were paired within experiments and the $t$-test employed to estimate the statistical significance of the differences.

\section{RESULTS}

The results are presented in Table 1. A gradual reduction in semen temperature after collection, as opposed to a more sudden reduction, did not significantly affect the fertilizing capacity of the semen in Experiment 1 although the data suggested that the period, during which fertile eggs were produced after a single insemination, was shorter with the semen that was subjected to a sudden temperature reduction. The absence of a detrimental effect of temperature shock during semen collection substantiates the results reported by De Silva (1963).

In Experiment 2 semen stored in the large vial, with an air-semen surface area of $154 \mathrm{~mm}^{2}$, exhibited greatly reduced fertilizing capacity compared with semen stored in the graduated vial (air-semen surface area of $50 \mathrm{~mm}^{2}$ ). It was suspected that this difference was due largely to the adverse effect of dehydration on the semen stored in the vial with the larger surface area, especially since the vials were not corked. The fertilizing capacity of semen stored in graduated vials in the Cryovac enclosure was similar to that reported by Proudfoot (1966) in which the same packaging technique was used.

In Experiment 3 the semen stored for $7 \mathrm{hr}$ at $10^{\circ} \mathrm{C}$ in the oxygen environment produced an average fertility over 12 days of $74.9 \%$ compared with $67.4 \%$ for the semen stored in air. This difference was significant at the $5 \%$ level and suggested that preventing dehydration, at least partially, by using a corked vial, is important in maintaining optimum fertilizing capacity.

In Experiment 4 bubbling oxygen through distilled water before using it as an atmosphere for fowl semen resulted in no significant improvement in fertility when $0.4 \mathrm{ml}$ of semen was stored at $10^{\circ} \mathrm{C}$ for a period of $7 \mathrm{hr}$ in large corked vials. Dehydration of semen, if it occurred, did not adversely affect the semen stored under the conditions of this experiment.

The effect of vial size, involving differences in atmospheric volume of gas and semen surface area exposed to the gas, was demonstrated in Experiment 5. The semen stored in the medium vial resulted in superior fertility compared with that in the large vial when stored at $5^{\circ} \mathrm{C}$ for $15 \mathrm{hr}$. 


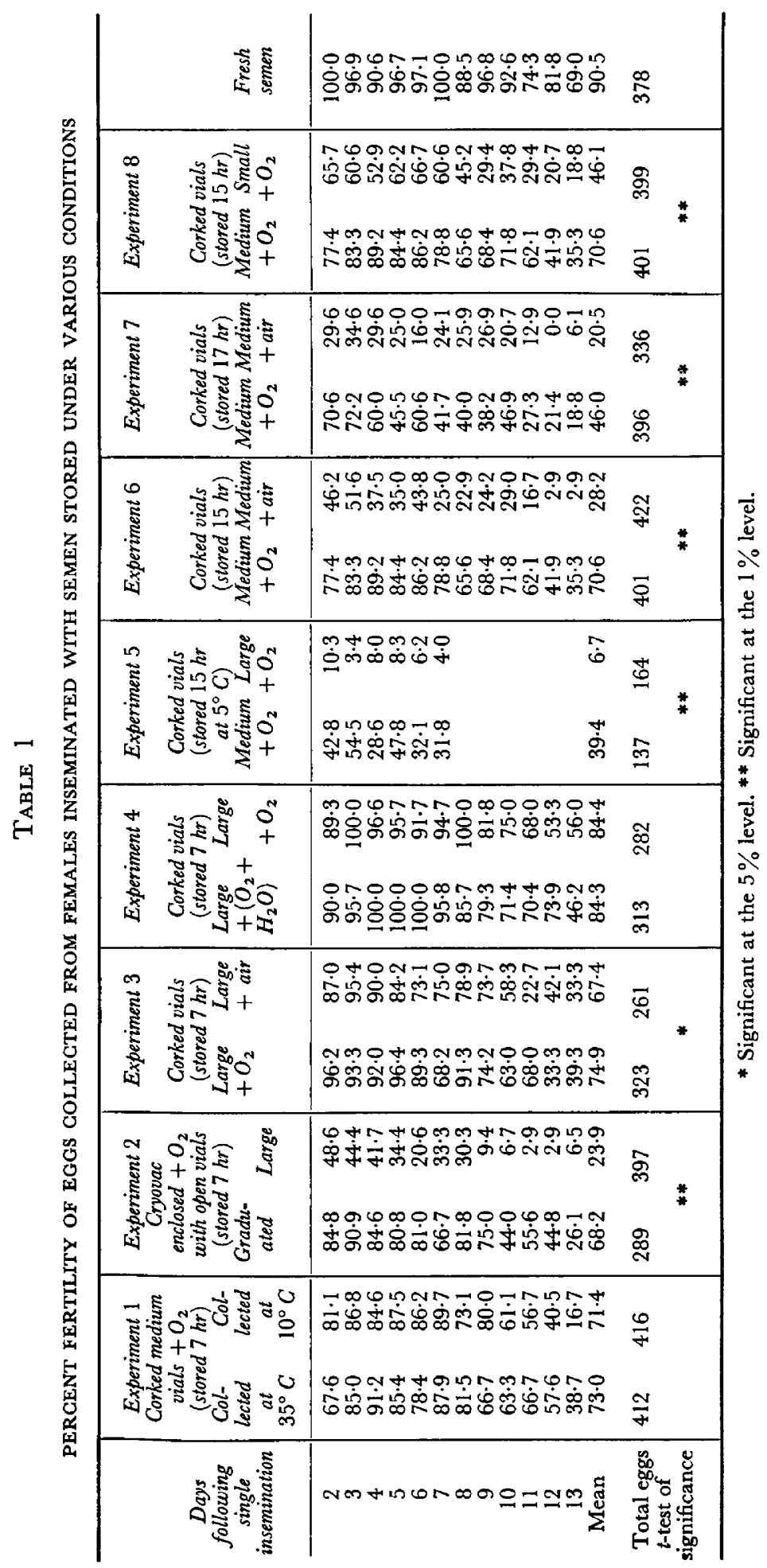


The beneficial effect of oxygen on the fertilizing capacity of fowl spermatozoa was demonstrated in Experiments 6 and 7. The difference in results was comparable with that of Experiment 3. However, increased storage time revealed better the difference in the effects of oxygen and air atmospheres. In Experiment 3 , storage period $7 \mathrm{hr}$, the fertilizing capacity of spermatozoa exhibited a difference of 7.5\%, whereas with the 15- and 17-hrstorage periods of Experiments 6 and 7 the difference in fertility was 52.4 and $25.5 \%$, respectively. Fertility may have been biased downward in Experiment 7 compared with Experiment 6 as insemination was conducted $1 \mathrm{hr}$ earlier in the day.

As the medium vial was demonstrated to be superior to the large vial in Experiment 5, Experiment 8 was conducted to compare the medium with a small vial. This experiment was carried out in conjunction with Experiment 6 and the overall fertility of semen stored in the medium vial was $70.6 \% \mathrm{com}$ pared with $46.1 \%$ in the small vial which had a reduced gas volume, reduced surface area and increased semen depth.

\section{DISGUSSION}

The results of the present series of experiments indicate that the fertilizing capacity of fowl semen maintained in the liquid state at $10^{\circ} \mathrm{C}$ is greatly enhanced by storage under oxygen rather than air.

The findings of Schindler \& Nevo (1962) may suggest an explanation for the present results if it is assumed that enhanced fertilizing capacity of the semen was dependent on a supply of oxygen to the spermatozoa sufficient to maintain aerobic metabolism.

The depth of the zone supplied with oxygen depends on the diffusion of oxygen into the liquid and the consumption of oxygen by the spermatozoa. At a certain depth $(d)$ these will balance and the oxygen concentration at $d$ will be zero. The depth $d$ may be calculated from the following formula (Schindler \& Nevo, 1962):

$$
d=\sqrt{\frac{2 D C}{a}}
$$

where: $D=$ diffusion co-efficient of oxygen,

$C=$ oxygen concentration above solution,

$a=$ rate of oxygen consumption by spermatozoa.

The depth of this zone is seen to be proportional to the square root of the oxygen concentration above the semen. Thus, if the oxygen concentration is increased from $21 \%$ (air) to $100 \%$ (pure oxygen) the depth of the zone is increased $2 \cdot 2$ times. This depth will be the same in the small, medium and large vials since it is independent of surface area.

The volume of semen exposed to the effects of oxygen was largest in the large vial due to the large surface area and thus the greatest enhancement of fertilizing capacity might have been expected in this vial. This was not the case and it is assumed that the harmful effect of dehydration, due to the large gas volume above the semen (Table 2), outweighed the beneficial effect of oxygen. 
Experiments 6 and 7 compared the fertilizing capacity of semen stored under air and oxygen in the medium vial where the volume of semen receiving a supply of oxygen could be expected to be $2 \cdot 24$ times as great under oxygen as under air. The increase in fertility (which paralleled this increased volume) was 2.5- and 2.2-fold in Experiments 6 and 7 respectively. Experiment 8 compared the fertilizing capacity of semen stored under oxygen in medium and small vials where the ratio of the volume of semen oxygenated, small/medium, was calculated to be $0 \cdot 53$. Again the ratio of the different fertility levels was found to be $0 \cdot 66$. The results of the three experiments above suggested that the fertilizing capacity of fowl semen was proportional to the volume of semen receiving sufficient oxygen for the spermatozoa to maintain aerobic metabolism.

TABLE 2

SPEGIFICATIONS OF VIALS USED IN EXPERIMENTS 2 TO 8 INGLUSIVE

\begin{tabular}{l|c|c|c}
\hline & \multicolumn{3}{|c}{ Vial } \\
\cline { 2 - 4 } & Small & Medium & Large \\
\hline Internal diameter (mm) & 8 & 11 & 16 \\
Height (mm) & 47 & 75 & 97 \\
$\begin{array}{l}\text { Surface area (air/semen) } \\
\text { interface (mm }{ }^{2} \text { ) }\end{array}$ & 50 & 95 & 154 \\
Maximum depth of semen (mm) & 12 & 8 & 6 \\
Approximate surface area of vial in & & & \\
$\quad$ contact with semen (mm $\left.{ }^{2}\right)$ & 300 & 260 & 300 \\
Gas volume over semen $\left(\mathrm{cm}^{3}\right)$ & $1 \cdot 8$ & 6.4 & 18.3 \\
\end{tabular}

Since the rate of oxygen consumption of fowl semen at $5^{\circ}$ and $10^{\circ} \mathrm{C}$ was not known, it was not possible to calculate the exact depth $d$ of the zone supplied with oxygen. Using a value calculated from oxygen consumption of fowl spermatozoa at $37^{\circ} \mathrm{C}$ (Goldberg \& Norman, 1961) the depth was likely to be approximately $2 \mathrm{~mm}$ and thus oxygen may not have diffused to the bottom of the semen in the medium vial which had a maximum depth of $8 \mathrm{~mm}$.

However, even if the numbers of spermatozoa in the zone supplied with oxygen were only a fraction of the total number, they still might have a profound effect on fertilizing capacity. Schindler, Nahari \& Bornstein (1963) have observed that insemination with as few as 25 million vigorously motile spermatozoa can give as good fertility results as when many times this number is used. Taneja \& Gowe (1962) reported that a difference among strains also exists, in that 40 million spermatozoa were required for optimum fertility with one strain, whereas another strain required 70 million.

It is known that fowl spermatozoa can retain their motility under anaerobic conditions if a sufficient supply of a reducing sugar (glucose, fructose, mannose) is present (Schindler \& Nevo, 1962). The concentration of reducing sugar, if any, present in the semen used for this investigation was not known. Sugar levels in fowl semen are quite low and are rapidly exhausted at room temperature (Schindler \& Nevo, 1962), so it is probable that in the present study there was no reducing sugar available in the semen to provide energy under anaerobic 
conditions. The spermatozoa would thus have to rely on internal (endogenous) reserves to maintain viability and motility.

It is generally accepted that carbohydrate is much more efficiently metabolized under aerobic than under anaerobic conditions. Fowl spermatozoa under anaerobic conditions may thus exhaust their reserves of carbohydrate more quickly, lose motility and, as a last resource, may metabolize structural elements of the cell, including the genetic material (DNA), and become infertile. The beneficial effects of oxygen in the present study are postulated to be due to increased efficiency of carbohydrate metabolism allowing the spermatozoon to maintain its viability longer and sparing the structure of the cell from enzymatic autolysis. The storage temperatures will, of course, lower the metabolic rate and also tend to increase the time during which the spermatozoa retain their fertilizing capacity.

\section{REFERENCES}

Burrows, W. H. \& Quins, J. P. (1937) The collection of spermatozoa from the domestic fowl and turkey. Poult. Sci. 16, 19.

DE SILva, P. L. G. (1963) Evidence for absence of an effect of temperature shock on fertilizing ability of fowl spermatozoa. 7. Reprod. Fert. 6, 371.

Goldberg, E. \& Norman, C. (1961) The metabolism of ejaculated spermatozoa from the fowl. $\mathcal{F}$. cell. comp. Physiol. 58, 175.

Parker, J. E., McKenzie, F. F. \& Kempster, H. L. (1942) Fertility in the male domestic fowl. Bull. Mo. agric. Exp. Sin, 347.

Proudfoot, F. G. (1966) The influence of oxygen and other gases on the fertilizing ability of fowl semen held for several hours at $10^{\circ} \mathrm{C}$ in Cryovac enclosures. Poult. Sci. 45, 443.

Schindler, H. \& Nevo, A. (1962) Reversible inactivation and agglutination of fowl and bull spermatozoa under anaerobic conditions. 7. Reprod. Fert. 4, 251.

Schindler, H., NAHARI, U. \& BoRnstein, S. (1963) The effect of sperm number and sperm concentration on fertility in artificial insemination of chickens. Israel 7. agric. Res. 13, 155.

TANEJA, G. C. \& GowE, R. S. (1962) Effect of varying doses of undiluted semen on fertility and hatchability in the domestic fowl. F. Reprod. Fert. 4, 161. 